\title{
REVUE
}

INTERNATIONALE
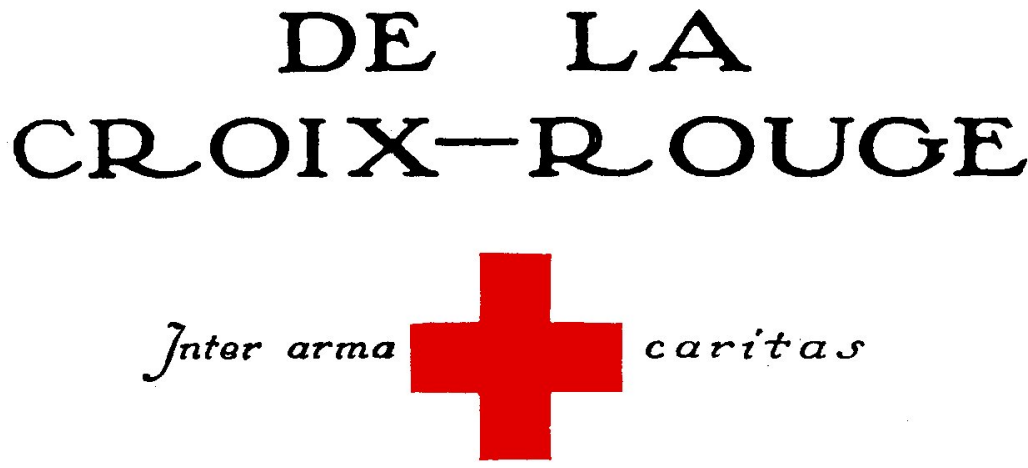

\section{BULLETIN INTERNATIONAL DES SOCIÉTÉS DE LA CROIX-ROUGE}

\section{T. LXI, $\mathbf{N}^{\circ} \mathbf{3} 30$ \\ SOMMAIRE :}

GRACE H. HUN'TER, diététicienne-chei de l'hôpital général Walter Reed, surintendante de l'Ecole de diététique de l'armée. Le rôle des diététiciennes dans l'armée américaine (hors texte).

SIDNEY H. BROWN, membre du seerétariat du Comité international.

La Convention internationale pour la sauvegarde de la vie humaine en mer, 1929 . . . . . . . . . . . . . .

Protection des populations civiles contre la Guerre CHIMQUe. - Opinions diverses sur la guerre chimique, 100. - Les cours de défense contre les gaz en Pologne, 105. Une séquelle de l'intoxication par les gaz, 106.

Chronique. Congrès du conseil international des femmes, 107. - Lutte contre le cancer, concours international, 107. Premier congrès international de microbiologie, 109.

Bibiographie. - Notice sur la vie et les travaux de M. Gustave Ador, 112. - La Convention de Genc̀ve de 1929 et l'immu. nisation des appareils sanitaires aériens, 113. - La guerra di ogni giorno, 118. - A travers les revues, 119.

Bulletin international des Cociétés de t.a Croix-Rouge (voir la $4^{\mathrm{e}} \mathrm{p}$. de la couverture)......... 


\section{Comité international de la Croix-Rouge}

à Genève

MM. Huber, Max, docteur en droit, juge et ancien président de la Cour permanente de justice internationale, président.

Werner, Georges, docteur en droit, professeur de droit public et administratif à l'Université de Genève, vice-président.

$M^{\text {me }}$ Chaponnière-Chaix, membre du Conseil international des femmes, vice-présidente.

MM. Palry, Georges, $D^{r}$, lieutenant-colonel et médecin de la $\mathrm{I}^{\mathrm{r}} \mathrm{e}$ division de l'armée suisse, vice-président.

de Haller, Rodolphe, banquier, trésorier.

Des Gouttes, Paul, docteur en droit, avocat.

Boissier, Edmond, colonel.

Bouvier, Bernard, professeur bonoraire de l'Université de Genève.

Chenevière, Jacques, homme de lettres.

Logoz, Paul, docteur en droit, professeur de droit pénal à l'Université de Genève.

Cramer, Lucien, docteur en droit.

Motta, Giuseppe, docteur en droit, conseiller fédéral.

de Meuron, Aloïs, avocat, ancien conseiller national.

Mlie Ferrière. Suzanne, secrétaire de l'International Migration Service.

MM. Audeoud, G. E., Dr, ancien médecin de division de l'armée suisse.

Dunant, Maurice, vice-président de la Croix-Rouge suisse.

\section{Membres honoraires}

MM. d'Espine, Adolphe, docteur-médecin, professeur honoraire de l'Université de Genève, associé étranger de l'Académie de médecine de Paris.

Barbey-Ador, Frédéric, ministre de Suisse à Bruxelles.

$M^{\text {me }}$ Ed. Frick-Cramer.

MM. Moynier, Adolphe, consul honoraire de Belgique.

Micheli, Horace, docteur ès-lettres, ancien conseiller national.

\section{REVUE INTERNATIONALE DE LA CROIX-ROUGE} ET BULLETIN DES SOCIETES NATIONALES

DE LA CROIX-ROUGE

Direction : M. Bernard Bouvier, membre du C.I. C.-R.

Rédaction : M. Etienne Clouzot.

M. Henri Reverdin.

Rédaction ct Administration :

Promenade du Pin, 1. - Téléphone : 51.365.

Adresse télégr. : Intercroixrouge.

\section{Adresses des Comités centraux.}

AFRIQUE DU SUD. - Croix-Rouge sud-africaine, 25, Belfast House, Market Street, Johannesburg.

ALBANIE. - Croix-Rouge albanaise, Tirana.

ALLEMAGNE. - Croix-Rouge allemande, Corneliusstrasse, 4 b, Berlin, W. 10.

ARGENTINE. - Croix-Rouge argentine, Cerrito, 1174, Buenos-Ayres.

AUSTRALIE. - Croix-Rouge australienne, 42-46, Latrobe Street, Melbourne.

AUTRICHE. - Croix-Rouge autrichienne (Bundesleitung der Oesterreichischen Gesell• schaft vom Roten Kreuze), Milchgasse, 1, Vienne I.

Voir suite couverture 3 . 


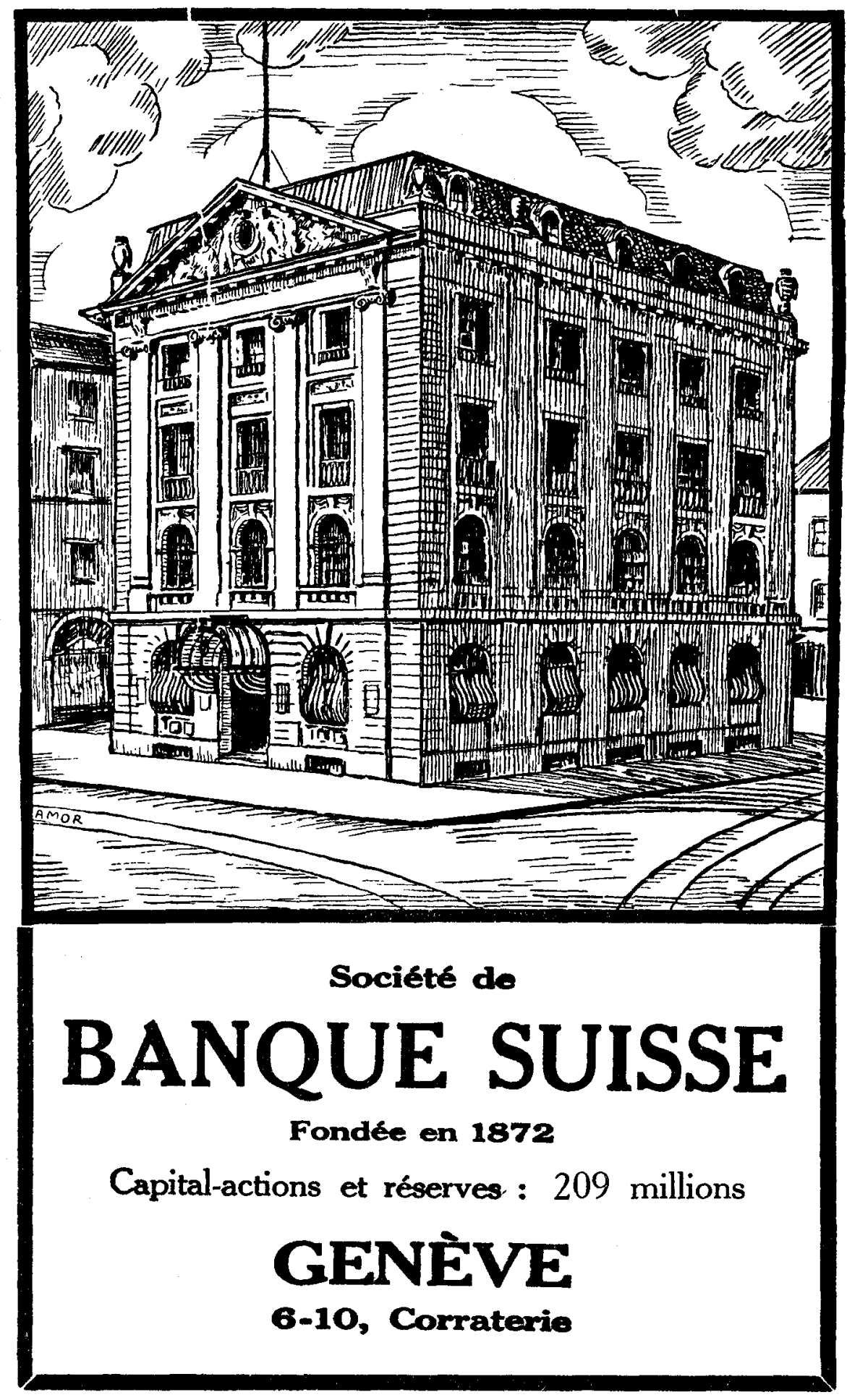




\section{Plus de deux cents}

travaux des spécialistes les plus en vue en matière de médecine des pays chauds, consignés dans la presse médicale du monde entier, font foi de $\mathrm{l}^{\text {' }}$ ACTION SOUVERAINE du

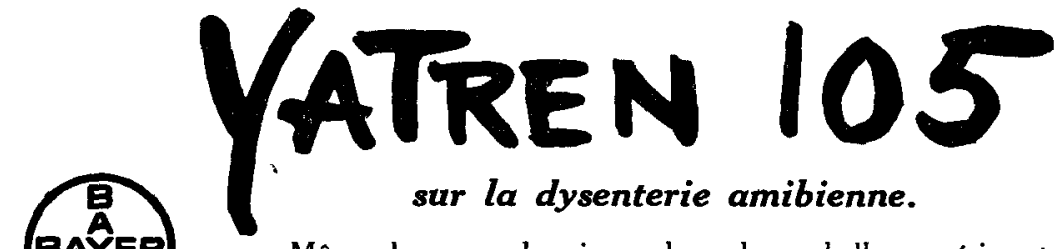

Même les cas chroniques les plus rebelles guérissent définitivement.

\section{G. FARBENINDUSTRIE AKTIENGESELLSCHAFT}

Dépt. pharm. scientif. "Rayer Neistorducius"

Leverkusen (Allemagne)

\section{REVUE \\ INTERNATIONALE DE L'ENFANT}

31, quai du Mont-Blanc, GENĖVE

Le numéro : 1 fr. suisse. 12 numéros (un an) : 10 fr. suisses

Sommaire du numéro de janvier 1930:

Le travail dans le champ de l'éducation et de l'assistance, par Sante de Sanctis, professeur à l'Université de Rome.

The Practical Value of Mental Hygiene to the Welfare of the Child, by Douglas $\Lambda$. Thom, M.D., State Division of Mental Hygiene, Boston.

Schwererziehbare Kinder. 1. von Dr H. Hanselmann, Privatdozent für Heilpädagogik an der Universität Zürich.

Chronique. - Nouvelles de l'U.I.S.E. - Bibliographie. -- Table des matières, T. VII et VIII. -- Illustrations. 